\title{
A CALCULUS APPROACH TO HYPERFUNCTIONS III
}

\author{
TADATO MATSUZAWA
}

\section{Introduction}

In the previous papers, [18] and [19], we have given some basis of a calculus approach to hyperfunctions. We have taken hyperfunctions with the compact support as initial values of the solutions of the heat equation. More precisely, let $A^{\prime}[K]$ be the space of analytic functionals supported by a compact subset $K$ of $R^{n}$ and let $E(x, t)$ be the $n$-dimensional heat kernel given by

$$
E(x, t)=(4 \pi t)^{-n / 2} \exp \left[\frac{-|x|^{2}}{4 t}\right], \quad t>0 .
$$

Then for $u \in A^{\prime}[K]$, the function $U(x, t)$ defined by

$$
U(x, t)=u_{y}(E(x-y, t)), \quad x \in R^{n}, \quad t>0,
$$

satisfies the heat equation in $R_{+}^{n+1}=\left\{(x, t) ; x \in R^{n}, t>0\right\}$ and we have

$$
u=\lim _{t \rightarrow 0_{+}} U(\cdot, t),
$$

where the limit is taken in the sense of (1.6) in Theorem 1.1. The main purpose of this paper is to give some applications to microlocal calculus on the basis of the results obtained so far. In Section 1, we recall some basic facts on hyperfunctions given in [18] and [19]. Furthermore we add to consider to characterize hyperfunctions in the whole space $R^{n}$ $\left(=\mathscr{B}\left(R^{n}\right)\right)$ in such a way that they are also initial values of the solutions of the heat equation and locally equivalent to analytic functionals with the compact support. At the end of Section 1 we shall recall the notions of the wave front sets following the results of [18] and [19]. In Section 2, we shall investigate the microlocal regularity of the solutions of pseudodifferential equations in hyperfunction spaces as well as in ultradistribution spaces. The fundamental tool is the local expression of hyperfunctions given in (2.4) and $(2.4)^{\prime}$, the latter is a special case of

Received January 9, 1989. 
(2.4). The formula (2.4) was given in [22] in Schwartz distribution case and in [19], (3.10) in hyperfunction case. We generalize this in the form (2.4) by means of the results prepared in Section 1 and we shall see it plays an important role in the mocrolocal calculus. The author would like to thank Y. Morimoto for the stimulating discussion with him.

\section{$\S 1$. Preliminaries}

We use general notations such as $|\alpha|=\alpha_{1}+\cdots+\alpha_{n}$ for a multiindex $\alpha=\left(\alpha_{1}, \cdots, \alpha_{n}\right)$ and $D^{\alpha}=D_{1}^{\alpha_{1}} \cdots D_{n}^{\alpha_{n}}, D_{j}=-i \partial / \partial x_{j}, j=1, \cdots, n$, etc.. Let $K$ be a compact subset of $R^{n}$ whose points are denoted by $x=$ $\left(x_{1}, \cdots, x_{n}\right)$. As in [19], let $A[K]$ denote the space of all real analytic functions in some neighborhood of $K . A^{\prime}[K]$ is the strong dual space of $A[K]$ and call its elements analytic functionals carried by $K$. Let $u \in$ $A^{\prime}[K]$ then for every complex neighborhood $\omega$ of $K$ we have

$$
|u(\varphi)| \leq C_{\omega} \sup _{\omega}|\varphi|, \quad \varphi \in A,
$$

where $A$ is the space of entire functions in $\Subset$. We refer to [19] the notion of Gevrey spaces $\mathscr{E}^{\mathscr{E}(s\}}$ and $\mathscr{E}^{\mathscr{E}\{s\}^{\prime}}, 1<s<\infty$, etc. The $n$-dimensional heat kernel is given by

$$
E(x, t)=(4 \pi t)^{-n / 2} \exp \left[-|x|^{2} / 4 t\right], \quad(x, t) \in R_{+}^{n+1} .
$$

Let $u \in A^{\prime}[K]$ the function

$$
U(x, t)=u_{y}(E(x, t))
$$

is well defined in $R_{+}^{n+1}$ and we shall call it the defining function of $u$.

Theorem 1.1 ([19], Theorem 2.1). (i) Let $u \in A^{\prime}[K]$ then $U(x, t) \in$ $C^{\infty}\left(R_{+}^{n+1}\right)$ and $U$ satisfies the following conditions:

$$
(\partial / \partial t-\Delta) U(x, t)=0 \quad \text { in } R_{+}^{n+1} ;
$$

For every $\varepsilon>0$ we have

$$
|U(x, t)| \leq C_{\varepsilon} \exp \left[(\varepsilon / t)-\operatorname{dis}(x, K)^{2} / 4 t\right] \quad \text { in } R_{+}^{n+1} ;
$$

$U(\cdot, t) \rightarrow u$ as $t \rightarrow 0_{+}$in the following sense:

$$
u(\varphi)=\lim _{t \rightarrow 0} \int_{\Omega} U(x, t) \varphi(x) d x, \quad \varphi \in A,
$$

where $\Omega$ is an arbitrary bounded neighborhood of $K$ in $R^{n}$ and $A$ is the space of entire functions in $\mathfrak{C}^{n}$. 
Conversely, every $C^{\infty}$-function defined in $R_{+}^{n+1}$ satisfying the conditions (1.4) and (1.5) can be expressed in the form (1.3) with unique element $u \in$ $A^{\prime}[K]$.

(ii) In case $u \in \mathscr{E}_{K}^{\{s\}^{\prime}}, 1<s<\infty$, the condition (1.5) of the above assertion is replaced by the following:

For any $\varepsilon, \delta>0$ we have

$$
|U(x, t)| \leq C_{\varepsilon, \delta} \exp \left[(\varepsilon / t)^{1 /(2 s-1)}-\operatorname{dis}\left(x, K_{\delta}\right)^{2} / 8 t\right] \quad \text { in } R_{+}^{n+1},
$$

where $K_{\delta}=\{x ; \operatorname{dis}(x, K) \leq \delta\}$.

(iii) In case $u \in \mathscr{E}_{K}^{\prime}$, the condition (1.5) is replaced by the following: There exists a non negative integer $N=N(u)$ such that

$$
|U(x, t)| \leq C_{\hat{\delta}} t^{-N} \exp \left[-\operatorname{dis}\left(x, K_{\delta}\right)^{2} / 8 t\right] \quad \text { in } R_{+}^{n+1} .
$$

We add the following characterization of the tempered distributions which is also useful.

Theorem 1.2. Let $u \in \mathscr{S}^{\prime}\left(R^{n}\right)$ then $U(x, t)=u_{y}(E(x-y, t)) \in C^{\infty}\left(R_{+}^{n+1}\right)$ and satisfies the following conditions:

$$
(\partial / \partial t-\Delta) U(x, t)=0 \quad \text { in } R_{+}^{n+1} ;
$$

There are positive constants $C, M$, and $N$ such that

$$
|U(x, t)| \leq C t^{-M}(1+|x|)^{N} \quad \text { in } R_{+}^{n+1} ;
$$

$U(x, t) \rightarrow u$ in $\mathscr{S}^{\prime}\left(R^{n}\right)$ as $t \rightarrow 0_{+}$, i.e.

$$
\lim _{t \rightarrow 0_{+}} \int U(x, t) \varphi(x) d x=u(\varphi), \quad \varphi \in \mathscr{P}\left(R^{n}\right) .
$$

Conversely, every $C^{\infty}$-function defined in $R_{+}^{n+1}$ satisfying the conditions (1.7) and (1.8) can be expressed in the form (1.3) with unique element $u \in$ $\mathscr{S}^{\prime}\left(R^{n}\right)$.

The proof of this theorem is obtained by the similar way as in Theorem 1.1 given in [18] and [19].

We can consider $A^{\prime}\left[K_{1}\right] \subset A^{\prime}\left[K_{2}\right]$ if $K_{1} \subset K_{2} \subset R^{n}$ and we set

$$
A^{\prime}=A^{\prime}\left(R^{n}\right)=\bigcup_{K} A^{\prime}[K] \text {. }
$$

We recall that the support of $u \in A^{\prime}\left(R^{n}\right)$ is the smallest compact set $K \subset R^{n}$ such that $u \in A^{\prime}[K]$. Now let $\Omega$ be a bounded open set in $R^{n}$. The space of hyperfunctions $\mathscr{B}(\Omega)$ is defined by 


$$
\mathscr{B}(\Omega)=A^{\prime}[\bar{\Omega}] / A^{\prime}[\partial \Omega] .
$$

We want to define hyperfunctions in $R^{n}$. Let $\Omega_{j}, j=1,2, \cdots$, be bounded open subsets of $R^{n}$ such that $R^{n}=\bigcup_{j=1}^{\infty} \Omega_{j}$. If $u_{j} \in A^{\prime}\left[\bar{\Omega}_{j}\right]$ and for all $j$, $k$ we have $u_{j}=u_{k}$ in $\Omega_{j} \cap \Omega_{k}$ (that is, $\left.\operatorname{supp}\left(u_{j}-u_{k}\right) \cap \Omega_{j} \cap \Omega_{k}=\varnothing\right)$ then for any bounded open set $\Omega \subset R^{n}$ there is a unique $u \in \mathscr{B}(\Omega)$ with $u=u_{j}$ in $\Omega \cap \Omega_{j}, j=1,2, \cdots$, by virtue of a localization theorem, [19], Theorem 2.4, ([7], Theorem 9.2.2) which implies the sheaf property of $\mathscr{B}(\Omega)$. We can therefore define a hyperfunction $u \in \mathscr{B}\left(R^{n}\right)$ as a collection of $u_{j} \in A^{\prime}\left[\bar{\Omega}_{j}\right]$ such that $u_{j}=u_{k}$ in $\Omega_{j} \cap \Omega_{k}, 1 \leq j, k<\infty$. The definition of $u \in \mathscr{B}\left(R^{n}\right)$ is independent of such bounded open $\Omega_{j}$ sets with $R^{n}=\bigcup \Omega_{j}$.

Definition 1.1. We call $U(x, t) \in C^{\infty}\left(R_{+}^{n+1}\right)$ an infra-exponential solution of the heat equation if it satisfies the heat equation in $R_{+}^{n+1}$ and for any compact subset $K$ of $R^{n}$ and $\varepsilon>0$ we have

$$
|U(x, t)| \leq C_{\varepsilon, K} e^{\varepsilon / t} \quad t>0, x \in K .
$$

THEOREM 1.3. Let $u \in \mathscr{B}\left(R^{n}\right)$, that is, let $u=\left\{u_{j}\right\}_{j=1}^{\infty}$ such that $u_{j} \in$ $A^{\prime}\left[\bar{\Omega}_{j}\right], u_{j}=u_{k}$ in $\Omega_{j} \cap \Omega_{k}, 1 \leq j, k<\infty$, where $\Omega_{j}$ are bounded open sets with $R^{n}=\cup \Omega_{j}$. Then there exists an infra-exponential solution $U(x, t)$ such that

$$
U(x, t)-U_{j}(x, t) \Longrightarrow 0 \text { as } t \longrightarrow 0_{+} \quad \text { in } \quad \Omega_{j}, j=1,2, \cdots,
$$

where $U_{j}(x, t)$ is the defining function of each $u_{j}$ and $\rightrightarrows$ means the weak uniform convergence in $\Omega_{j}$.

Conversely, let $U(x, t)$ be an infra-exponential solution, then there exists a unique $u \in \mathscr{B}\left(R^{n}\right)$ satisfying $U(\cdot, t) \rightarrow u$ as $t \rightarrow 0_{+}$in the sense of (1.12), that is, for any bounded open sets $\Omega_{j}$ with $R^{n}=\bigcup_{j=1}^{\infty} \Omega_{j}$, there exist $u_{j} \in A^{\prime}\left[\bar{\Omega}_{j}\right]$ whose defining functions $U_{j}(x, t)$ satisfy (1.12).

Proof. Necessity. For simplicity we shall prove the one dimensional case of $n=1$. Take $\Omega_{j}=\left\{x \in R^{1} ;|x|<r_{j}=\sum_{i=1}^{j}(1 / i)\right\}, j=1,2, \cdots$, then obviously we have $R^{1}=\cup \Omega_{j}$. Assume that there are given $u_{j} \in A^{\prime}\left[\bar{\Omega}_{j}\right]$ such that $u_{j}=u_{k}$ in $\Omega_{j}=\Omega_{j} \cap \Omega_{k}, 1 \leq j<k<\infty$. Let $U_{j}(x, t)$ be the defining function of $u_{j}$ :

$$
U_{j}(x, t)=u_{j y}(E(x-y, t)) \quad \text { in } R_{+}^{n+1}, \quad j=1,2, \cdots .
$$

We shall construct infra-exponential functions $V_{j}(x, t)$ such that 


$$
\begin{gathered}
(\partial / \partial t-\Delta) V_{j}(x, t)=0 \quad \text { in } R_{+}^{n+1} ; \\
V_{j} \Longrightarrow 0 \text { as } t \longrightarrow 0_{+} \quad \text { in } \quad R^{n} \backslash \partial \Omega_{j}, \quad j=1,2, \cdots ; \\
\left|U_{j+1}-U_{j}+V_{j}-V_{j+1}\right| \leq C 2^{-j} \exp \left[\varepsilon_{j} \mid t\right], \quad t>0,
\end{gathered}
$$

where $C$ is a positive constant independent of $j$ and $\varepsilon_{j} \rightarrow 0$ as $j \rightarrow \infty$. Furthermore for any $M>0$ there exists a number $j_{M}$ and a constant $C_{M}$ such that

$$
\begin{gathered}
j \geq j_{M} \Longrightarrow\left|U_{j+1}-U_{j}+V_{j}-V_{j+1}\right| \leq C_{M} 2^{-j} \exp \left[-1 / C_{M} t\right], \\
t>0, \quad|x| \leq M .
\end{gathered}
$$

Then it follows from (1.16) and (1.13) that the limit

$$
U=\lim _{j \rightarrow \infty}\left(U_{j}-V_{j}\right)=U_{j}-V_{j}+\sum_{k=j}^{\infty}\left(U_{k+1}-U_{k}+V_{k}-V_{k+1}\right)
$$

exists and satisfies the heat equation in $R_{+}^{n+1} . U(x, t)$ is an infra-exponential solution by $(1.16)$ and $U(x, t)-U_{j}(x, t) \Rightarrow 0$ in $\Omega_{j}$. It remains to construct such $V_{j}(x, t)$. We take $V_{1}(x, t) \equiv 0$. Assuming that we have $V_{j}(x, t)$ we construct $V_{j+1}$. Let $v_{j} \in A^{\prime}\left[\partial \Omega_{j}\right]$ be the initial value of $V_{j}(x, t)$ and put

$$
u_{j+1}-u_{j}+v_{j}=g_{j} \in A^{\prime}\left[\bar{\Omega}_{j+1}\right]
$$

Then we have

$$
\operatorname{supp}\left[g_{j}\right] \subset\left[-r_{j+1},-r_{j}\right] \cup\left[r_{j}, r_{j+1}\right]
$$

and

$$
U_{j+1}(x, t)-U_{j}(x, t)-V_{j}(x, t)=\int E(x-y, t) g_{j}(y) d y,
$$

where we have used the integral in the distribution sense. For the sake of the simplicity we assume supp $\left[g_{j}\right] \subset\left[r_{j}, r_{j+1}\right]$. By using the Taylor expansion of $E(x-y, t)$ at $y=r_{j+1}$ the right hand side of (1.17) is written by

$$
\sum_{\alpha \geq 0} \frac{1}{\alpha !} E^{(\alpha)}\left(x-r_{j+1}, t\right) \int\left(r_{j+1}-y\right)^{\alpha} g_{j}(y) d y
$$

Taking $N$ a large number determined later, we set

$$
V_{j+1}(x, t)=\sum_{\alpha=0}^{N} \frac{1}{\alpha !} E^{(\alpha)}\left(x-r_{j+1}, t\right) \int\left(r_{j+1}-y\right)^{\alpha} g_{j}(y) d y .
$$


If we apply the estimates of the derivatives of $E(x, t)$ given in [19], Proposition 1.1 and the inequality (1.1) for $g_{j}$, we have

$$
\begin{aligned}
\mid U_{j+1} & -U_{j}+V_{j}-V_{j+1} \mid \\
\leq & \left|\sum_{\alpha \geq N+1} \frac{1}{\alpha !} E^{(\alpha)}\left(x-r_{j+1}, t\right) \int\left(r_{j+1}-y\right)^{\alpha} g_{j}(y) d y\right| \\
& \leq C_{j} \sum_{\alpha \geq N+1} C^{\alpha} t^{-(1+\alpha) / 2} \alpha !^{-1 / 2} \exp \left[-\left(x-r_{j+1}\right)^{2} / 8 t\right](2 / j)^{\alpha} \\
& \leq C_{j}^{\prime} t \exp [\varepsilon / t] \exp \left[-\left(x-r_{j+1}\right)^{2} / 8 t\right] \sum_{\alpha \geq N+1}(2 C / j \sqrt{\varepsilon})^{\alpha}, \quad \varepsilon>0 .
\end{aligned}
$$

Taking $\varepsilon=\varepsilon_{j}=(4 C / j)^{2}$, this is majorated by the quantity of the form

$$
C_{j} t^{-1 / 2} \exp \left[\varepsilon_{j} / t\right] \exp \left[-\left(x-r_{j+1}\right)^{2} / 8 t\right] \sum_{\alpha \geq N+1}(1 / 2)^{\alpha} .
$$

If we take $N$ sufficiently large for each $j \geq 1$, we obtain the estimate (1.15). The other estimates are obtained similarly.

Sufficiency. We take a function $\psi_{j}(x, t) \in C^{\infty}\left(R_{+}^{n+1}\right) \cap L^{\infty}\left(R_{+}^{n+1}\right)$ such that

$$
\begin{array}{ll}
\psi_{j}(x, t)=0 & \text { for }\left\{x^{2}+t^{2}>j^{2}, t>0\right\} \\
\psi_{j}(x, t)=1 & \text { for }\left\{x^{2}+4 t^{2} \leq j^{2}, t>0\right\}, j=1,2, \cdots .
\end{array}
$$

(cf. [7]), Corollary 1.4.11.)

Then by [14], Theorem 2.27 there exist ultradistributions $\widetilde{\psi_{j}} U \in \mathscr{D}^{\{2\}^{\prime}}\left(R^{n+1}\right)$ such that $\widetilde{\psi_{j} U}=\psi_{j} U$ for $t>0$ and $\widetilde{\psi_{j} U}=0$ for $t<0$. We define ultradistributions $\tilde{F}_{j} \in \mathscr{E}^{\{2\}^{\prime}}\left(R^{n+1}\right)$ and $\tilde{V}_{j} \in \mathscr{D}^{\{2\}^{\prime}}\left(R^{n+1}\right)$ by

$$
\tilde{F}_{j}(x, t)=(\partial / \partial t-\Delta) \widetilde{\psi_{j}} U(x, t), \quad \tilde{V}_{j}(x, t)=E * \tilde{F}_{j},
$$

where we consider $E *$ as a pseudodifferential operator from $\mathscr{E}^{\{2\}^{\prime}}\left(R^{n+1}\right)$ to $\mathscr{D}^{\{2\}^{\prime}}\left(R^{n+1}\right)$, (cf. [17]). If we set

$$
U_{j}(x, t)=\widetilde{\psi_{j}} \widetilde{U}(x, t)-\tilde{V}_{j}(x, t), \quad t>0,
$$

then taking notice of the support of $\tilde{F}_{j}$ we see $U_{j}(x, t)$ is an infra-exponential solution of the heat equation and

$$
U_{j}(x, t) \longrightarrow 0 \text { as } t \longrightarrow 0_{+} \text {in } R^{n} \backslash \bar{\Omega}_{j} .
$$

Thus by Theorem 1.1, there is a unique element $u_{j} \in A^{\prime}\left[\bar{\Omega}_{j}\right]$ such that $U_{j}(\cdot, t) \rightarrow u_{j}$ as $t \rightarrow 0_{+}$in the sense of (1.6). We have $U(\cdot, t)-U_{j}(\cdot, t) \rightarrow 0$ as $t \rightarrow 0_{+}$in $\Omega_{j}$ since $\tilde{V}_{j}(\cdot, t) \rightrightarrows 0$ as $t \rightarrow 0_{+}$in $\Omega_{j}$, which is verified by 
using the pseudolocal property of the operator $E *$, (cf. [17]). This completes the proof of Theorem 1.3.

Let $u \in A^{\prime}$, then the defining function $U(x, t)=u_{y}(E(x-y, t))$ is extensible as an entire analytic function in $\mathbb{C}^{n}$ for each fixed $t>0$. We will consider the asymptotic property of $U(z, t)$ as $t \rightarrow 0$ for $z=x+$ $i \xi \in \mathbb{E}^{n}$.

TheOREm 1.4. (i) Let $u \in A^{\prime}[K]$ then we have the estimate of the form

$$
\begin{gathered}
|U(x+i \xi, t)| \leq C_{\varepsilon} \exp \left[\left(\xi^{2}+\varepsilon(1+|\xi|)-\operatorname{dis}(x, K)^{2}\right) / 4 t\right], \\
x+i \xi \in \mathfrak{S}^{n}, \quad t>0, \varepsilon>0 .
\end{gathered}
$$

(ii) Let $u \in \mathscr{E}^{\{s\}^{\prime}}, 1<s<\infty$, then we have

$$
\begin{aligned}
|U(x+i \xi, t)| \leq & C_{\varepsilon, \delta} \exp \left[\xi^{2} / 4 t+\varepsilon\left((1 / t)^{1 /(2 s-1)}\right)\right. \\
& \left.+(|\xi| \mid t)^{1 / s}-\operatorname{dis}\left(x, K_{\delta}\right)^{2} / 8 t\right], \quad x+i \xi \in \mathfrak{C}^{n}, t>0 .
\end{aligned}
$$

(iii) Let $u \in \mathscr{S}^{\prime}\left(R^{n}\right)$ then there are positive constants $C, M$ and $N$ such that

$$
\begin{gathered}
|U(x+i \xi, t)| \leq C t^{-M}(1+|x|+|\xi|)^{N} \exp \left[\xi^{2} / 4 t\right], \\
x+i \xi \in \mathfrak{C}^{n}, \quad t>0 .
\end{gathered}
$$

Now remember the definitions of the wave front sets.

Definition 1.2 ([19], Definition 5.1). Let $u \in A^{\prime}\left(R^{n}\right)$. We denote by $W F_{A}(u), W F_{\{s\}}(u), 1<s<\infty$, and $W F(u)$ respectively the complement in $T^{*}\left(R^{n}\right) \backslash 0$ of the set of $\left(x_{0}, \xi_{0}\right)$ such that there is a neighborhood $V$ of $x_{0}-i \xi_{0}$ in $\overleftarrow{\complement}^{n}$ and there are positive constants $C$ and $c$ satisfying

$$
\begin{array}{ll}
|U(z, t)| \leq C \exp \left[\xi_{0}^{2} / 4 t-c / t\right], & t>0, z=x+i \xi \in V, \\
|U(z, t)| \leq C \exp \left[\xi^{2} / 4 t-(c / t)^{1 / s}\right], & t>0, z \in V,
\end{array}
$$

and there are positive constants $C_{N}, N=0,1, \cdots$, such that

$$
|U(z, t)| \leq C_{N} t^{N} \exp \left[\xi^{2} / 4 t\right], \quad t>0, z \in V,
$$

respectively.

By this definition we have obviously the following inclusions:

$$
W F(u) \subset W F_{\{s\}}(u) \subset W F_{A}(u), \quad u \in A^{\prime}, 1<s<\infty .
$$

Let $\Omega$ be a bounded open set of $R^{n}$. Let $\Gamma$ be an open cone in $R^{n} \backslash\{0\}$ and set for $\gamma>0$

$$
W=\left\{z \in \mathfrak{S}^{n} ; \mathscr{R}_{e} z \in \Omega, \mathscr{I}_{m} z \in \Gamma,\left|\mathscr{I}_{m} z\right|<\gamma\right\} .
$$


Let $f=f(z)$ be holomorphic in $W$, then it is well known that there is unique boundary value $b_{\Gamma}(f) \in \mathscr{B}(\Omega)$ of $f(z)$ from $\Gamma$. We have shown this fact via heat kernel in [19], Theorem 5.2 and Theorem 5.3.

Theorem 1.5 ([19], Theorem 5.4). Let $u \in A^{\prime}$ and $\left(x_{0}, \xi_{0}\right) \in T^{*}\left(R^{n}\right) \backslash 0$. Then

(i) $\left(x_{0}, \xi_{0}\right) \notin W F_{A}(u)$ if and only if there are open convex cones $\Gamma_{1}, \cdots$, $\Gamma_{N} \subset\left\{\xi \in R^{n} ;\left\langle\xi, \xi_{0}\right\rangle\langle 0\}\right.$, bounded open neighborhood $Z$ of $x_{0}$ in $\mathbb{E}^{n}, u_{0}$ holomorphic in $Z, f_{j}(z)$ holomorphic in $Z \cap\left\{R^{n}+i \Gamma_{j}\right\}, j=1, \cdots, N$, so that

$$
u=u_{0}+\sum_{1}^{N} b_{\Gamma j}\left(f_{j}\right) \quad \text { in } Z \cap R^{n} .
$$

(ii) $\left(x_{0}, \xi_{0}\right) \notin W F_{\{s\}}(u), 1<s<\infty$, if and only if $u_{0} \in \mathscr{E}^{\{s\}}\left(Z \cap R^{n}\right)$ in the above expression (1.23).

(iii) $\left(x_{0}, \xi_{0}\right) \notin W F(u)$ if and only if $u_{0} \in \mathscr{E}\left(Z \cap R^{n}\right)$ in the above expression (1.23).

Conversely, Theorem 1.5 gives Definition 1.2, in other words, the following description of the wave front sets.

Theorem 1.6. Let $u \in A^{\prime}$ and $\left(x_{0}, \xi_{0}\right) \in T^{*}\left(R^{n}\right) \backslash 0$. Then

(i) $\left(x_{0}, \xi_{0}\right) \notin W F_{A}(u)$ if and only if there exist positive constants $C, c$ and $\varepsilon$ such that

$$
\begin{aligned}
|U(x-i \omega, t)| \leq C \exp [(1-c) / 4 t], & t>0, \omega \in S^{n-1}, \\
\left|\omega-\omega_{0}\right|+\left|x-x_{0}\right|<\varepsilon, & \omega_{0}=\frac{\xi_{0}}{\left|\xi_{0}\right|} \in S^{n-1} .
\end{aligned}
$$

(ii) $\left(x_{0}, \xi_{0}\right) \notin W F_{\{s\}}(u), 1<s<\infty$, if and only if there exist positive constants $C, c$ and $\varepsilon$ such that

$$
\begin{aligned}
|U(x-i \omega, t)| \leq C \exp \left[1 / 4 t-(c / t)^{1 / s}\right], & t>0, \omega \in S^{n-1}, \\
\left|\omega-\omega_{0}\right|+\left|x+x_{0}\right|<\varepsilon, & \omega_{0}=\frac{\xi_{0}}{\left|\xi_{0}\right|} \in S^{n-1} .
\end{aligned}
$$

(iii) $\left(x_{0}, \xi_{0}\right) \notin W F(u)$ if and only if there exist positive constants $\varepsilon$ and $C_{N}, N=0,1,2, \cdots$, such that

$$
\begin{array}{cl}
|U(x-i \omega, t)| \leq C_{N} t^{N} \exp [1 / 4 t], & t>0, N=0,1,2, \cdots, \\
\left|\omega-\omega_{0}\right|+\left|x-x_{0}\right|<\varepsilon, & \omega_{0}=\frac{\xi_{0}}{\left|\xi_{0}\right|} \in S^{n-1} .
\end{array}
$$


As was pointed out by J.M. Bony [2], we can easily verify that these definitions of the wave front sets are equivalent to those of $\mathrm{L}$. Hörmander in case $u$ is a Schwartz distribution.

\section{§2. Pseudodifferential equations}

First we remember a class of pseudodifferential operators treated in [5] [16] and [17].

Definition 2.1. Let $\Omega$ be an open subset of $R^{n}$ and let $-\infty<m$ $<\infty ; 0 \leq \delta<\rho \leq 1 ; \sigma \geq 1 . \quad S_{\rho, \hat{o}, \sigma}^{m}\left(\Omega \times R^{n}\right)$ denotes the set of all $a(x, \xi) \epsilon$ $C^{\infty}\left(\Omega \times R^{n}\right)$ such that for every compact subset $K$ of $\Omega$ there are positive constants $C_{0}, C_{1}$ and $B$ such that

$$
\sup _{x \in K}\left|a_{(\beta)}^{(\alpha)}(x, \xi)\right| \leq\left. C_{0} C_{1}^{|\alpha+\beta|}\left|\alpha ! \beta !^{\sigma}\right| \xi\right|^{m-\rho|\alpha|}, \quad|\xi| \geq B|\alpha|^{\theta},
$$

where $a_{(\beta)}^{(\alpha)}(x, \xi)=\partial_{\xi}^{\alpha} D_{x}^{\beta} a(x, \xi), \theta=\max (1 / \rho, \sigma /(1-\delta))$.

We associate with such a symbol $a(x, \xi)$ a pseudodifferential operator $a(x, D)$ as follows:

$$
a(x, D) u(x)=(2 \pi)^{-n} \iint e^{i\langle x-y, \xi\rangle} a(x, \xi) u(y) d y d \xi, \quad u \in \mathscr{D}(\Omega) .
$$

It is well known that $a(x, D)$ is extended to a continuous mapping from $\mathscr{E}^{\prime}(\Omega)$ into $\mathscr{D}^{\prime}(\Omega)$, (cf. [6]). We call $a(x, D)$ an analytic pseudodifferential operator if $a(x, \xi) \in S_{1,0,1}^{m}\left(\Omega \times R^{n}\right)$, i.e. in case $\rho=1, \delta=0$ and $\sigma=1$ in (2.1). Let $a(x, D)$ be an analytic pseudodifferential operator and let $u \in A^{\prime}[K], K \in \Omega$. Then $a(x, D) u(x) \in \mathscr{B}(\Omega)$ is well defined as follows:

Take a function $\chi \in C_{0}^{\infty}(\Omega)$ such that $\chi=1$ in a neighborhood of $K$. Let $U(x, t)$ be the defining function of $u$. Then for every $\varphi \in A$, we have

$$
\int \chi a(x, D)(\chi U)(x, t) \varphi(x) d x=\int \chi(x) U(x, t)^{t} a(x, D)\left(\chi_{\varphi}\right)(x) d x, \quad t>0 .
$$

By virtue of the analytic pseudolocal property of ${ }^{t} a(x, D)$ proved in [5]

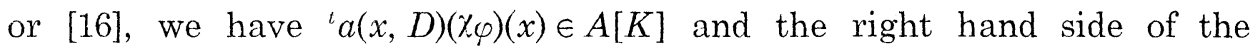
above equation tends to $\left\langle u,{ }^{t} a(x, D)\left(\chi_{\varphi}\right)(x)\right\rangle$ as $t \rightarrow 0$, (cf. (1.6)). We define $\chi_{a}(x, D) u$ by the formula

$$
\left\langle\chi_{a}(x, D) u, \varphi\right\rangle=\left\langle u,{ }^{t} a(x, D) \chi_{\varphi}\right\rangle, \quad \varphi \in A .
$$

Then we have $\gamma a(x, D) u \in A^{\prime}[\operatorname{supp} \gamma]$. On the other hand we have

$$
(1-\chi) a(x, D) u(x)=\int(1-\chi(x)) K(x, y) u(y) d y \in C^{\infty}(\Omega),
$$


where $K(x, y)$ is the kernel of $a(x, D)$ which is real analytic in $\Omega \times \Omega \backslash \Delta$, $\Delta=\{(x, x) ; x \in \Omega\}$, (cf. [5], [16]). We define

$$
a(x, D) u=\chi a(x, D) u+(1-\chi) a(x, D) u \in \mathscr{B}(\Omega) .
$$

We can easily see that this definition is independent of such a function $\chi$ and agrees with the usual definition of $a(x, D) u \in \mathscr{D}^{\prime}(\Omega)$ when $u \in \mathscr{E}^{\prime}(\Omega)$. We can see also $a(x, D) u$ is analytic in $\Omega \backslash K$. By this process we can say that we have in the oscillatory sense

$$
\begin{aligned}
a(x, D) u(x) & =(2 \pi)^{-n} \int e^{i\langle x, \xi\rangle} a(x, \xi) \hat{u}(\xi) d \xi, \\
& =\lim _{t \downarrow 0}(2 \pi)^{-n} \int e^{i\langle x, \xi\rangle} a(x, \xi) \hat{U}(\xi, t) d \xi, \\
& =\lim _{t \downarrow 0}(2 \pi)^{-n} \pi^{-n / 2} \int e^{i\langle x, \xi\rangle-4 t|\xi|^{2}} a(x, \xi) \hat{u}(\xi) d \xi .
\end{aligned}
$$

An important tool in microlocal analysis is the following local expression of $a(x, D) u$.

Theorem 2.1. (i) Let $a(x, \xi) \in S_{1,0,1}^{m}\left(\Omega \times R^{n}\right)$ and $u \in A^{\prime}[K], \quad K \subset \Omega$. Then for any $x_{0} \in \Omega$ and $\varepsilon>0,0<\varepsilon \ll 1$, we have

$$
\begin{aligned}
a(x, D) u= & (2 \pi)^{-n} \iint_{\substack{\left|\beta-x_{0}\right| \leq 2 \varepsilon \\
|\xi| \geq B}} u_{y}\left(\exp \left[i\langle x-y, \xi\rangle-(\beta-y)^{2}|\xi| / 2\right]\right) \\
& \times a(x, \xi)(|\xi| / 2 \pi)^{n / 2} d \beta d \xi+w_{\varepsilon}(x),
\end{aligned}
$$

where $w_{\varepsilon}(x) \in \mathscr{B}(\Omega)$ and $w_{\varepsilon}(x)$ is analytic in $\left|x-x_{0}\right|<\varepsilon$. As a special case with $a \equiv 1$ we have

$$
\begin{aligned}
u(x)= & (2 \pi)^{-n} \iint_{\substack{\left|\beta-x_{0}\right| \leq 2 \varepsilon \\
|\xi| \geq B}} u_{y}\left(\exp \left[i\langle x-y, \xi\rangle-(\beta-y)^{2}|\xi| / 2\right]\right) \\
& \times(|\xi| / 2 \pi)^{n / 2} d \beta d \xi+w_{\varepsilon}(x)
\end{aligned}
$$

where $w_{\varepsilon}(x) \in \mathscr{B}(\Omega)$ and $w_{\varepsilon}(x)$ is analytic in $\left|x-x_{0}\right|<\varepsilon$.

(ii) Let $a(x, \xi) \in S_{\rho, \delta, \sigma}^{m}\left(\Omega \times R^{n}\right)$ and $u \in \mathscr{E}^{[\tilde{\theta}\}^{\prime}}(\Omega)$, where we assume the number $\tilde{\theta} \equiv \max (1 / \rho, \sigma+\delta / \rho)>1$. Then we have $a(x, D) u \in \mathscr{D}^{\{\tilde{\theta}\}^{\prime}}(\Omega)$ and the same formula as in (2.4) holds with $w_{\varepsilon}(x) \in \mathscr{D}^{(\tilde{\theta})^{\prime}}(\Omega)$ and $w_{\varepsilon}(x)$ is in $\mathscr{E}^{\mathscr{E}[\tilde{\theta}\}}\left(\left|x-x_{0}\right|<\varepsilon\right)$.

(iii) Let $a(x, \xi) \in S_{\rho, \delta}^{m}\left(\Omega \times R^{n}\right)$ a usual $C^{\infty}$-symbol of the type $(\rho, \delta)$ and $u \in \mathscr{E}^{\prime}(\Omega)$. Then we have $a(x, D) u \in \mathscr{D}^{\prime}(\Omega)$ and the formula (2.4) is valid with $w_{\varepsilon}(x) \in \mathscr{D}^{\prime}(\Omega)$ and $w_{\varepsilon}(x)$ is in $\mathscr{E}\left(\left|x-x_{0}\right|<\varepsilon\right)$. 
Proof of (i). Let $u \in A^{\prime}[K], K \subset \Omega$. Since

$$
(|\xi| / 2 \pi)^{n / 2} \int_{R^{n}} \exp \left[-(\beta-y)^{2}|\xi| / 2\right] d \beta=1
$$

we have in the oscillatory sense as in (2.3)

$$
\begin{aligned}
a(x, D) u(x)= & (2 \pi)^{-n} \iint_{|\xi| \geq B} u_{y}\left(\exp \left[i\langle x-y, \xi\rangle-(\beta-y)^{2}|\xi| / 2\right]\right) a(x, \xi) \\
& \times(|\xi| / 2 \pi)^{n / 2} d \beta d \xi+u_{y}(g(x, y)),
\end{aligned}
$$

where

$$
g(x, y)=(2 \pi)^{-n} \iint_{|\xi| \geq B} e^{i\langle x-y, \xi\rangle} a(x, \xi) d \xi .
$$

The function $g(x, y)$ is analytic in $\Omega \times R^{n}$. Furthermore for any fixed $x_{0} \in \Omega$ and $\varepsilon>0$ sufficiently small so that $\left\{\left|y-x_{0}\right|<\varepsilon\right\} \subset \Omega$ the function

$$
F(x, y)=\iint_{\substack{\left|\beta-x_{0}\right|<2 \varepsilon \\|\xi| \leq B}} \exp \left[i\langle x-y, \xi\rangle-(\beta-y)^{2}|\xi| \mid 2\right](|\xi| / 2 \pi)^{n / 2} a(x, \xi) d \xi d \beta
$$

is analytic in the region $D=\left\{(x, y) \in R^{n} \times R^{n} ;\left|x-x_{0}\right|<\varepsilon, y \in R^{n}\right\}$. In fact it is clear for $\left|x-x_{0}\right|<\varepsilon$ and $\left|y-x_{0}\right|<2 \varepsilon$ since $|\beta-y|>0$. In case where $\left|x-x_{0}\right|<\varepsilon$ and $\left|y-x_{0}\right|>3 \varepsilon / 2$ we devide the $\beta$-integral into the regions (a) $|\beta-y| \geq \delta$ and (b) $|\beta-y| \leq \delta, 0<\delta \ll \varepsilon$. In order to treat the case (b), we make the almost analytic extension of the symbol $a(x, \xi)$ with respect to $\xi$ as follows:

$$
\tilde{a}(x, \xi+i \eta)=\sum_{|\alpha|+1 \leq|\xi| / B} \partial_{\xi}^{\alpha} a(x, \xi)(i \eta)^{\alpha} / \alpha ! .
$$

Then we have the estimates of the form:

$$
\begin{gathered}
\left|\partial_{x}^{\mu} \tilde{a}(x, \xi+i \eta)\right| \leq C_{0} C_{1}^{|\mu|} \mu !|\xi|^{m}, \\
\left|\partial_{x}^{\mu}\left(\partial / \partial \bar{\zeta}_{j}\right) \tilde{a}(x, \xi+i \eta)\right| \leq C_{0} C_{1}^{|\mu|} \mu !(|\eta||| \xi \mid)^{c|\xi|}|\xi|^{m},
\end{gathered}
$$

where $\zeta=\xi+i \eta,|\eta| \leq c^{\prime}|\xi|,|\xi| \geq B, 0<c^{\prime} \ll 1, c=B^{-1}$. By using the Stokes formula on the $(n+1)$-chain defined by

$$
\zeta=\xi+i \eta, \quad|\xi| \geq B, \quad \eta=t(x-y)|\xi|, \quad 0 \leq t \leq \gamma, \quad 0<\gamma \ll 1,
$$

we can estimate the $D_{x}^{\mu}$-derivatives of

$$
\begin{aligned}
F_{\delta}(x, y)= & \iint_{\substack{\left|\beta-x_{0}\right| \geq 2 \varepsilon \\
|\xi| \geq B|\leq \delta\\
| \beta-y \mid \leq \delta}} \exp \left[i\langle x-y, \xi\rangle-(\beta-y)^{2}|\xi| / 2\right](|\xi| / 2 \pi)^{n / 2} \\
& \times a(x, \xi) d \xi d \beta
\end{aligned}
$$


by those of the integral defined by making the complex deformation in (2.8):

$$
\left\{\begin{array}{c}
\xi \longrightarrow \zeta=\xi+i \gamma(x-y)|\xi|, \quad 0<\gamma \ll 1, \\
d \xi \longrightarrow d \zeta=(1+i \gamma\langle x-y, \xi \| \xi \mid\rangle) d \xi_{1} \wedge \cdots \wedge d \xi_{n}
\end{array}\right.
$$

and by those of

$$
\begin{gathered}
D_{x}^{\mu} \int_{0}^{\gamma} d t \iint_{\substack{\left|\beta-x_{0} \geq 2 \delta\\
\right| 1 \geq B \\
|\beta-y| \leq \delta}} \exp \left[i\langle x-y, \xi+i t(x-y)|\xi|\rangle-(\beta-y)^{2}|\xi| / 2\right] \\
\quad \times \sum_{j}\left(\partial / \partial \bar{\zeta}_{j}\right) \tilde{a}(x, \zeta)(|\xi| / 2 \pi)^{n / 2}(1+i t\langle x-y, \xi /|\xi|\rangle) d \beta d \xi
\end{gathered}
$$

with the remainder of analytic function (integral on $|\xi|=B, 0<t<\gamma$ ). We remark that we have $|x-y|>\varepsilon / 2\left(\left|x-x_{0}\right|<\varepsilon,\left|y-x_{0}\right|>3 \varepsilon / 2\right)$ in this case. Then it is clear the integral defined in (2.9) is analytic in $(x, y)$. As for (2.10) it turns out that we have to estimate essentially the integrals of the form

$$
\int_{0}^{r} d t \int_{|\xi| \geq B} e^{-t(x-y)^{2}|\xi|}|\xi|^{m+|\mu|} t^{c|\xi|} d \xi,
$$

where $|x-y| \geq \varepsilon / 2$ and $c>0$. We devide this integral into two parts:

$$
\mathrm{I}=\int_{0}^{r} d t \int_{B \leq|\xi| \leq\left(m_{+}+|\mu|+n\right) / c} \cdots d \xi
$$

and

$$
\mathrm{II}=\int_{0}^{r} d t \int_{|\xi| \geq\left(m_{+}+|\mu|+n\right) / c} \cdots d \xi, \quad\left(m_{+}=\max (0, m)\right) .
$$

Since $0 \leq t \leq \gamma \ll 1$, we can easily verify that we have the estimates of the form

$$
\mathrm{I} \leq C^{|\mu|+1}|\mu|^{|\mu|}
$$

Furthermore we have

$$
\mathrm{II} \leq \int_{0}^{r} d t \int_{|\xi| \geq\left(m_{+}+|\mu|+n\right) / c} \exp [-t \varepsilon|\xi| / 2](t|\xi|)^{m_{+}+|\mu|} t^{c|\xi|-|\mu|-m+} d \xi .
$$

Since we have $c|\xi|-|\mu|-m_{+} \geq n$ in this case, we have also the estimates of the form

$$
\mathrm{II} \leq C^{|\mu|+1}|\mu| !
$$

by making use of the transformation of the $\xi$-variable. We can also 
estimate $D_{y}^{\mu} F_{\delta}(x, y)$ similarly. In conclusion, we have obtained the formula

$$
\begin{aligned}
a(x, D) u(x)= & (2 \pi)^{-n} \iint_{\substack{\left|\beta-x_{0}\right| \leq 2 \varepsilon \\
|\xi| \geq B}} u_{y}\left(\exp \left[i\langle x-y, \xi\rangle-(\beta-y)^{2}|\xi| / 2\right]\right) \\
& \times a(x, \xi)(|\xi| \mid 2 \pi)^{n / 2} d \beta d \xi+w_{\varepsilon}(x)
\end{aligned}
$$

where $w_{\varepsilon}(x) \in \mathscr{B}(\Omega)$ and $w_{\varepsilon}(x)$ is analytic in $\left|x-x_{0}\right|<\varepsilon$.

Proof of (ii). Almost the same process as in the proof of (i) works well. We only give the sketch of the proof. In this case, we make the almost analytic extension of $a(x, \xi)$ as follows:

$$
\tilde{a}(x, \xi+i \eta)=\sum_{|\alpha|+1 \leq|\xi| \rho / B} \partial_{\xi}^{\alpha} a(x, \xi)(i \eta)^{\alpha} / \alpha ! .
$$

Then the estimation corresponding to (2.6) and (2.7) are given by

$$
\begin{gathered}
\left|\partial_{x}^{\mu} \tilde{a}(x, \xi+i \eta)\right| \leq C_{0} C_{1}^{|\mu|} \mu !^{\sigma}|\xi|^{m+\delta|\mu|}, \\
\left|\partial_{x}^{\mu}(\partial / \partial \bar{\zeta}) \tilde{a}(x, \xi+i \eta)\right| \leq C_{0} C_{1}^{|\mu|} \mu !^{o}\left(\left.|\eta||| \xi\right|^{\mid \rho}\right)^{c|\xi|}|\xi|^{m+\delta|\mu|},
\end{gathered}
$$

where $\zeta=\xi+i \eta, \quad|\eta| \leq c^{\prime}|\xi|^{o}, \quad|\xi| \geq B, \quad 0<c^{\prime} \ll 1, \quad c=B^{-1}$. We take $(n+1)$-chain defined by

$$
\zeta=\xi+i \eta, \quad|\xi| \geq B, \quad \eta=t(x-y)|\xi|^{\rho}, \quad 0 \leq t \leq r, \quad 0<\gamma \ll 1 .
$$

The complex deformation of the $\xi$-contour corresponding to (2.9) is as follows:

$$
\left\{\begin{array}{l}
\xi \longrightarrow \zeta=\xi+i \gamma(x-y)|\xi|^{\rho}, \quad 0<\gamma \ll 1, \\
d \xi \longrightarrow d \zeta=\left(1+i \gamma \rho|\xi|^{\rho-2}\langle x-y, \xi\rangle\right) d \xi_{1} \wedge \cdots \wedge d \xi_{n} .
\end{array}\right.
$$

The integral corresponding to I and II are evaluated by the quantity of the form

$$
C^{|\mu|+1} \mu !^{\tilde{\theta}}, \quad \tilde{\theta}=\max (1 / \rho, \sigma+\delta / \rho) .
$$

So we can see that the integral on $(2.9)^{\prime}$ is a Gevrey function in $(x, y)$ of the order $\max (1 / \rho, \sigma+\delta / \rho)$.

Proof of (iii). The core of the proof also lies in the reasoning as in (b) of the part (i). We can't shift the $\xi$-integral into the complex contour in this case. However, for $x \neq y$, by making use of the formula

$$
\begin{aligned}
F(x, y)= & \iint_{\substack{\left|\beta-x_{0}\right| \geq 2 \varepsilon \\
|\xi| \geq B}}\left(x_{j}-y_{j}\right)^{-k}\left(D_{\xi j}^{k} e^{i\langle x-y, \xi\rangle) \exp \left[-(\beta-y)^{2}|\xi| / 2\right]}\right. \\
& \times a(x, \xi)(|\xi| / 2 \pi)^{n / 2} d \xi d \beta
\end{aligned}
$$


for any $k \geq 0$ and for some $j, x_{j}-y_{j} \neq 0$, we conclude finally the function $F(x, y)$ is in $C^{\infty}(D), D=\left\{(x, y) ;\left|x-x_{0}\right|<\varepsilon, y \in R^{n}\right\}$.

In the following we shall give the microlocal version of the results on pseudolocal property given in [16], [17] and [18].

Theorem 2.2. (i ) Let $a(x, \xi) \in S_{1,0,1}^{m}\left(\Omega \times R^{n}\right)$ and $u \in A^{\prime}[K], K \subset \Omega$. Then we have

$$
W F_{A}(a(x, D) u) \subset W F_{A}(u) .
$$

(ii) Let $a(x, \xi) \in S_{\rho, \delta, \sigma}^{m}\left(\Omega \times R^{n}\right)$ with $\tilde{\theta}=\max (1 / \rho, \sigma+\delta / \rho)>1$ and let $u \in \mathscr{E}^{[\tilde{\theta}\}^{\prime}}(\Omega)$. Then we have

$$
\left.W F_{\{\tilde{\theta}\}^{\prime}}(a, x, D) u\right) \subset W F_{\{\tilde{\theta}\}^{\prime}}(u) .
$$

(iii) Let $a(x, \xi) \in S_{\rho, \delta}^{m}\left(\Omega \times R^{n}\right)$ and $u \in \mathscr{E}^{\prime}(\Omega)$. Then we have

$$
W F(a(x, D) u) \subset W F(u) .
$$

Proof. (i) Let $u \in A^{\prime}$ and $\left(x_{0}, \xi_{0}\right) \notin W F_{A}(u)$. From the expression

$$
U(x-i \omega, t)=(4 \pi t)^{-n / 2} \exp [1 / 4 t] u_{y}\left(\exp \left[\left(-(x-y)^{2}+2 i\langle x-y, \omega\rangle\right) / 4 t\right]\right)
$$

we have the equivalent condition to (1.21):

$$
\begin{gathered}
\left|u_{y}\left(\exp \left[\left(-(x-y)^{2}+2 i\langle x-y, \omega)\right\rangle \mid 4 t\right]\right)\right| \leq C \exp [-c / t], \quad t>0, \\
\left|\omega-\omega_{0}\right|+\left|x-x_{0}\right|<\delta, \quad \omega_{0}=\xi_{0} /\left|\xi_{0}\right| \in S^{n-1} .
\end{gathered}
$$

Putting $\xi^{\prime}=\omega / 2 t$ in (2.14) and rewriting $\xi^{\prime}$ as $\xi$ to get the estimate of the form

$$
\begin{gathered}
\mid u_{y}\left(\exp \left[i\langle x-y, \xi\rangle-(x-y)^{2}|\xi| / 2\right]\right) \leq C \exp [-c|\xi|] \\
\left|x-x_{0}\right| \leq \delta, \quad \xi \in V\left(\xi_{0}\right)
\end{gathered}
$$

where $\delta$ is a small positive number and $V\left(\xi_{0}\right)$ denotes a cone neighborhood of $\xi_{0}$. For $\xi_{1} \in R^{n} \backslash 0,\left\langle\xi_{1}, \xi_{0}\right\rangle<0$, let $\Gamma_{\xi_{1}}$ be a closed cone neighborhood of $\xi_{1}$ such that

$$
\left\langle\eta, \xi_{1}\right\rangle>0,\left\langle\eta, \xi_{0}\right\rangle<0 \quad \text { for any } \eta \in \Gamma_{\xi_{1}} .
$$

Let $\Gamma_{\xi_{1}}^{*}$ be the dual cone of $\Gamma_{\xi_{1}}$, i.e.

$$
\langle\eta, \xi\rangle>0 \quad \text { on } \Gamma_{\xi_{1}} \times \Gamma_{\xi_{1}}^{*} .
$$

We can choose $\xi_{1}, \cdots, \xi_{N}$ so that

$$
R^{n} \backslash 0=V \cup \stackrel{\circ}{\Gamma}_{\xi_{1}}^{*} \cup \cdots \cup \stackrel{\circ}{\Gamma}_{\xi_{N}}^{*} .
$$


Now return to the local expression (2.4):

$$
\begin{aligned}
a(x, D) u(x)= & (2 \pi)^{-n} \iint_{\substack{\left|\beta-x_{0}\right| \leq 2 \xi \\
|\xi| \geq B}} u_{y}\left(\exp \left[i\langle x-y, \xi\rangle-(\beta-y)^{2}|\xi| / 2\right]\right) \\
& \times a(x, \xi)(|\xi| / 2 \pi)^{n / 2} d \beta d \xi+w_{\varepsilon}(x)
\end{aligned}
$$

where $w_{\varepsilon}(x)$ is analytic in $\left|x-x_{0}\right|<\varepsilon$. We take the following cut-off symbols $\chi_{j}$ such that

$$
\operatorname{supp} \chi_{j} \subset \Gamma_{\xi_{j}}^{*}, \quad \sum_{1}^{N} \chi_{j}=1 \quad \text { on } R^{n} \backslash V .
$$

Furthermore, we can assume there are positive constants $C_{0}$ and $C_{1}$ satisfying

$$
\left|\partial_{\xi}^{\alpha} \chi_{j}(\xi)\right| \leq C_{0} C_{1}^{|\alpha|} \alpha !|\xi|^{-|\alpha|} \quad \text { if }|\xi| \geq B|\alpha|, j=1, \cdots, N .
$$

Such a method of the construction of $\chi_{j}$ is given in [25], Chapter 5, Lemma 1.4. Considering $a(x, \xi) \chi_{j}(\xi)$ are analytic symbols and choosing $2 \varepsilon$ smaller than $\delta$ in (2.15), we have

$$
\begin{aligned}
a(x, D) u(x)= & u_{0}(x)+\sum_{1}^{N}(2 \pi)^{-n} \iint_{\substack{\left|\beta-x_{0}\right| \leq 2 \varepsilon \\
|\xi|>B}} u_{y}(\exp [i\langle x-y, \xi\rangle \\
& \left.-|\xi|(\beta-y)^{2} / 2\right](|\xi| / 2 \pi)^{n / 2} a(x, \xi) \chi_{j}(\xi) d \xi d \beta \\
\equiv & u_{0}(x)+\sum_{1}^{N} u_{j}(x) .
\end{aligned}
$$

Assumption (2.15) yields that $u_{0}(x)$ is analytic in $\left|x-x_{n}\right|<\varepsilon$. By using Theorem 5.2 and Theorem 5.3 of [19] we can see that each $u_{j}$ is the boundary value of

$$
\begin{aligned}
f_{j}(z)= & (2 \pi)^{-n} \iint_{\substack{\left|\beta-x_{0}\right| \leq 2 \xi \\
|\hat{\xi}| \geq B}} u_{y}\left(\exp \left[i\langle z-y, \xi\rangle-|\xi|(\beta-y)^{2} / 2\right]\right. \\
& \times(|\xi| / 2 \pi)^{n / 2} a(z, \xi) \chi_{j}(\xi) d \xi d \beta,
\end{aligned}
$$

which is holomorphic in $\tilde{Z} \cap\left(R^{n}+i \Gamma_{\xi_{j}}\right)$ with a small complex neighborhood $\tilde{Z}$ of $x_{0}$. Then by Theorem 1.5, we conclude that $\left(x_{0}, \xi_{0}\right) \notin W F_{A}$ $(a(x, D) u)$.

Proof of (ii). In case $\sigma=1$ the proof can be obtained by the same way as in the part (i). The estimate (2.15) is merely replaced by

$$
\begin{aligned}
\left|u_{v}\left(\exp \left[i\langle x-y, \xi\rangle-(x-y)^{2}|\xi| \mid 2\right]\right)\right| \leq & C \exp \left[-c|\xi|^{1 / \tilde{\theta}}\right], \\
& \left|x-x_{0}\right| \leq \delta, \quad \xi \in V\left(\xi_{0}\right) .
\end{aligned}
$$


In case $\sigma>1$ the symbol $a(x, \xi)$ is not analytic in $x$. So we make the device such that

$$
a(x, \xi)=\sum_{0}^{N} a_{j}(x, \xi), \quad\left|x-x_{0}\right| \leq \delta, \quad \xi \in R^{n} .
$$

Here $a_{j}(x, \xi)$ is analytic in $z \in Z \cap\left\{R^{n}+i \Gamma_{j}\right\}, j=0,1, \cdots, N$, where $Z$ is a small complex neighborhood of $x_{0}$ and $a_{j}(x, \xi)$ satisfies the same condition as in (2.2) in $Z \cap\left\{R^{n}+i \bar{\Gamma}_{j}\right\}$. This is possible by making use of Fourier inversion formula in $x$.

The proof of the part (iii) is obtained also by using the formula (2.4), though we omit the details.

Theorem 2.3. (cf. [18], Theorem 3.2.) Let $a(x, \xi) \in S_{1,0,1}^{m}\left(\Omega \times R^{n}\right)$ and $\left(x_{0}, \xi_{0}\right) \in T^{*}(\Omega) \backslash 0$. Assume that there is a cone neighborhood $V$ of $\xi_{0}$ and there are positive constants $c$ and $B$ and $-\infty<m^{\prime}<\infty$ such that

$$
|a(x, \xi)| \geq c|\xi|^{m^{\prime}}, \quad x \in \Omega, \quad|\xi| \geq B .
$$

Also assume that for any compact subset $K$ of $\Omega$, there are positive constants $C_{0}$ and $C_{1}$ such that

$\left(\mathrm{H}_{2}\right) \quad\left|a_{(\beta)}^{(\alpha)}(x, \xi)\right| \leq C_{0} C_{1}^{|\alpha+\beta|} \alpha ! \beta !|a(x, \xi)||\xi|^{-|\alpha|}, \quad x \in K, \quad \xi \in V, \quad|\xi| \geq B|\alpha|$.

Then if $u \in A^{\prime}[K], K \in \Omega$, and $\left(x_{0}, \xi_{0}\right) \notin W F_{A}(a(x, D) u)$, we have $\left(x_{0}, \xi_{0}\right) \notin$ $W F_{A}(u)$

Proof. First we follow the same steps as in the proof of [5], Theorem 3.1. Determine recursively the symbols $b_{j}$ by means of the relations

$$
b_{0}(x, \xi) a(x, \xi)=1, \quad \xi \in V,|\xi| \geq B
$$

and for $j=1,2, \cdots$

$$
b_{j}(x, \xi) a(x, \xi)=-\sum_{1 \leq|\alpha| \leq j} \frac{1}{\alpha !} \partial_{x}^{\alpha} a(x, \xi) D_{\xi}^{\alpha} b_{j-|\alpha|}(x, \xi), \quad \xi \in V, \quad|\xi| \geq B .
$$

Then for any compact subset $K$ of $\Omega$ there are constants $C_{0}$ and $C_{1}$ such that

$$
\begin{aligned}
\sup _{x \in K}\left|b_{j(\beta)}^{(\alpha)}(x, \xi)\right| & \leq C_{0} C_{1}^{|\alpha+\beta|+j} \alpha ! \beta !(1+|\xi|)^{-m^{\prime}-|\alpha|-j}, \\
& \xi \in V, \quad|\xi| \geq B(|\alpha|+j), \quad j=0,1, \cdots .
\end{aligned}
$$

Let $V_{0}$ and $V_{1}$ be any fixed closed convex cones such that $\xi_{0} \in V_{0} \Subset V_{1}$ $\subset V$. Prepare the functions $\varphi_{j}(\xi) \in C^{\infty}\left(R^{n}\right)$ satisfying for $j=0,1, \cdots$ 


$$
\begin{array}{lll}
0 \leq \varphi_{j}(\xi) \leq 1, \quad \xi \in R^{n}, & \text { and } \\
\varphi_{j}=0 & \text { if }|\xi| \leq 2 R \max (j, 1) & \text { or } \xi \notin V_{1} \\
\varphi_{j}=1 & \text { if }|\xi| \leq 3 R \max (j, 1) & \text { and } \xi \in V_{0}
\end{array}
$$

$$
\left|D^{\alpha} \varphi_{j}(\xi)\right| \leq(C / R)^{|\alpha|} \quad \text { if }|\alpha| \leq 2 j
$$

Take $R>B$ and set

$$
b(x, \xi)=\sum_{j=0}^{\infty} \varphi_{j}(\xi) b_{j}(x, \xi) .
$$

Then we have $b(x, \xi) \in S_{1,0,1}^{-m^{\prime}}\left(\Omega \times R^{n}\right)$. Take $h \in C_{0}^{\infty}(\Omega)$ such that $h \equiv 1$ in a neighborhood of $K$. The symbol of the operator $r(x, D)=b(x, D) h a(x, D)$ satisfies

$$
r(x, \xi)+1+q(x, \xi), \quad|\xi| \geq B, \quad \xi \in V_{0}
$$

for $x$ in a neighborhood $\Omega^{\prime}$ of $K, K \subset \Omega^{\prime} \subset \Omega$, and $q(x, \xi)$ satisfies the estimate of the form

$$
\sup _{x \in \Omega^{\prime}}\left|D_{x}^{\tau} q(x, \xi)\right| \leq C_{0} C_{1}^{|\tau|} \tau !|\xi|^{m-m^{\prime}} e^{-c|\xi|}, \quad \xi \in V_{0} .
$$

This means $q(x, D)$ is an analytic regularizer in the direction $V_{0}$. We remark that $h a(x, D) u(x) \in A^{\prime}$ is well defined. Now return to the local expression (2.4) and (2.4)'. For sufficiently small $\varepsilon$ we have

$$
\begin{aligned}
b(x, D) h a(x, D) u(x) & =(2 \pi)^{-n} \iint_{\substack{\left|\beta-x_{0}\right| \leq 2 \varepsilon \\
|\xi| \geq B}} u_{y}(\exp [i\langle x-y, \xi\rangle \\
& \left.\left.-(\beta-y)^{2}|\xi| / 2\right]\right) r(x, \xi)(|\xi| / 2 \pi)^{n / 2} d \beta d \xi+w_{\varepsilon}(x) .
\end{aligned}
$$

As in the proof of Theorem 2.2, choose a system of closed convex cones $\Gamma_{1}, \cdots, \Gamma_{N}$ such that

$$
\begin{aligned}
& \left\langle\eta, \xi_{0}\right\rangle<0 \quad \text { for any } \eta \in \Gamma_{j}, \\
& R^{n} \backslash 0=V_{0} \cup \stackrel{\circ}{\Gamma}_{1}^{*} \cup \cdots \cup \stackrel{\circ}{\Gamma}_{N}^{*} \quad \text { and } \quad \xi_{0} \in \Gamma_{j}^{*}, j=1, \cdots, N .
\end{aligned}
$$

Then we have

$$
\begin{aligned}
& u(x)=b(x, D) h a(x, D) u(x)+\sum_{j=1}^{N}(2 \pi)^{-n} \iint_{\substack{\left|\beta-x_{0}\right| \leq 2 \varepsilon \\
|\xi| \geq B}} u_{y}(\cdots)(r(x, \xi)-1) \\
& \times(|\xi| / 2 \pi)^{n / 2} \chi_{j}(\xi) d \beta d \xi+v_{\varepsilon}(x),
\end{aligned}
$$

where $v_{\varepsilon}(x)$ is analytic in $\left|x-x_{0}\right|<\varepsilon$ and $\chi_{j}(\xi)$ are the cut-off symbols as in the proof of Theorem 2.2. We can see each term of the summation in the right hand side is a boundary value of holomorphic function 
defined in $\left\{z=x+i y ;\left|x-x_{0}\right|<\varepsilon, y \in \Gamma_{j}, 0<|y|<\gamma, 0<\gamma \ll 1\right\}$. Thus we conclude that $\left(x_{0}, \xi_{0}\right) \notin W F_{A}(u)$ by Theorem 1.5 and Theorem 2.2.

Theorem 2.4 (cf. [17], Theorem 2.2). Let $a(x, \xi) \in S_{\rho, \delta, \sigma}^{m}\left(\Omega \times R^{n}\right), 0 \leq \delta$ $<\rho \leq 1, \sigma \geq 1$, and $\left(x_{0}, \xi_{0}\right) \in T^{*}(\Omega) \backslash 0$. Assume that there is a cone neighborhood $V$ of $\xi_{0}$ and there are positive constants $c$ and $B$ and $-\infty<m^{\prime}<\infty$ such that $\left(\mathrm{H}_{1}\right)$ in Theorem 2.3 holds. Also assume that for any compact subset $K$ of $\Omega$, there are positive constants $C_{0}$ and $C_{1}$ such that

$\left(\mathrm{H}_{2}\right)^{\prime}$

$$
\begin{aligned}
\left|a_{(\beta)}^{(\alpha)}(x, \xi)\right| \leq C_{0} C_{1}^{|\alpha+\beta|} \alpha ! \beta !^{\alpha}|a(x, \xi)||\xi|^{-\rho|\alpha|+\delta|\beta|}, & \\
& x \in K, \quad|\xi| \geq B, \quad \xi \in V .
\end{aligned}
$$

Furthermore assume $\theta \equiv \max (1 / \rho, \sigma /(1-\delta))>1$. Then if $u \in \mathscr{E}^{[\theta\}^{\prime}}(\Omega)$ and $\left(x_{0}, \xi_{0}\right) \notin W F_{\{\theta\}}(a(x, D) u)$, then $\left(x_{0}, \xi_{0}\right) \notin W F_{\{\theta\}}(u)$.

Remark. Generally we have

$$
\tilde{\theta}=\max (1 / \rho, \sigma+\delta / \rho) \leq \theta=\max (1 / \rho, \sigma /(1-\delta)),
$$

where the equality holds if and only if $\tilde{\theta}=\theta=1 / \rho$.

Proof of Theorem 2.4. We can obtain merely a weaker conclusion by only the same process of the proof of Theorem 2.3 in case $\theta>1$ as was seen in a series of papers [5], [16] and [17] which treated the ordinary hypoelliptic problems. However, summing up the method used in the proof of Theorem 2.3 and those of [16], [17] and [24], the proof of the theorem will be accomplished. It needs rather long and tedious calculation, so we shall give an outline of the proof.

First determine recursively the symbols $b_{j}(x, \xi), j=0,1, \cdots$, by means of the relations (2.17) and (2.18). We assume the existence of the following cut-off symbol $g(\xi)$ which was constructed in [25], Chapter 5, Lemma 1.4. Let $\xi_{0} \in V_{0} \subset V_{1} \subset V$ be closed convex cones in $R^{n} \backslash\{0\}$. There exists a function $g(\xi)$ such that

$$
0 \leq g(\xi) \leq 1, \quad \xi \in R^{n} ;
$$

(2.26) there exist positive constants $C_{0}$ and $C_{1}$ such that

$$
\left|\partial_{\xi}^{\alpha} g(\xi)\right| \leq C_{0} C_{1}^{|\alpha|} \alpha !|\xi|^{-|\alpha|} \quad \text { if } \quad|\hat{\xi}| \geq B|\alpha| ;
$$

$$
g(\xi)=1 \text { if } \xi \in V_{0},|\xi| \geq 1 \text { and } g(\xi)=0 \text { if } \xi \notin V_{1},|\xi| \geq B \text {. }
$$

We set 


$$
b(x, \xi)=g(\xi) \sum_{j=0}^{N_{0}} b_{j}(x, \xi),
$$

where the number $N_{0}$ is taken sufficiently large so that $(\rho-\delta) N_{0}-m+$ $m^{\prime}>2 n+1$ for later convenience. Then we have $b(x, \xi) \in S_{\rho, \delta, \sigma}^{-m^{\prime}}\left(\Omega \times R^{n}\right)$. Take a function $h \in \mathscr{D}^{\mid \theta\}}(\Omega)$ such that $h=1$ in a neighborhood of supp $u$ $\subset \Omega$. By the formula (2.5) with $0<\varepsilon \ll 1$ we have

$$
\begin{aligned}
& b(x, D) h a(x, D) u(x) \\
& =(2 \pi)^{-n} \iint_{\substack{\left|\beta-x_{0}\right| \leq 2 \varepsilon \\
|\xi| \geq B}}(h a u)_{y}\left(\exp \left[i\langle x-y, \xi\rangle-(\beta-y)^{2}|\xi| / 2\right]\right) \\
& \quad \times(|\xi| / 2 \pi)^{n / 2} b(x, \xi) d \xi d \beta+w_{\varepsilon}(x),
\end{aligned}
$$

where $w_{\varepsilon}(x) \in \mathscr{E}^{\{\theta\}}\left(\left|x-x_{0}\right|<\varepsilon\right)$. By assumption the first term in the right hand side is in $\mathscr{E}^{\mathscr{E}(\theta\}}\left(\left|x-x_{0}\right|<\varepsilon\right), 0<\varepsilon \ll 1$. On the other hand, by using the results of [16] and [17], we can write

$$
\begin{aligned}
& b(x, D) h a(x, D) u(x) \\
& =(2 \pi)^{-n} \iint_{\substack{\left|\beta-x_{0}\right| \leq 2 \varepsilon \\
|\xi| \geq B}} u_{y}\left(\exp \left[i\langle x-y, \xi\rangle-(\beta-y)^{2}|\xi| / 2\right]\right) \\
& \quad \times(|\xi| / 2 \pi)^{n / 2} R^{N_{0}}(x, \xi) d \xi d \beta+v_{\varepsilon}(x),
\end{aligned}
$$

where $v_{\varepsilon}(x) \in \mathscr{E}^{\mathscr{f}(\theta\}}\left(\left|x-x_{0}\right|<\varepsilon\right)$ and the symbol $R^{N_{0}}(x, \xi)$ satisfies the condition of the form

$$
\begin{gathered}
\left|\partial_{x}^{\beta} \partial_{\xi}^{r} R^{N_{0}}(x, \xi)\right| \leq C_{0} C_{1}^{|\beta+\gamma| \gamma ! \beta ! \sigma|\xi|^{m++n-\rho|\gamma|} \sum_{\tau \leq \beta}\left(\begin{array}{l}
\beta \\
\tau
\end{array}\right)|\tau|^{|\delta| \tau \mid}|\xi|^{|\delta| \beta-\tau|+\delta 2| \tau \mid} .} \\
m_{+}=\max \left(m-m^{\prime}, 0\right), \quad x \in \Omega^{\prime} \subset \Omega, \quad|\xi| \geq B+1, \quad\left(\operatorname{supp} u \subset \Omega^{\prime}\right) .
\end{gathered}
$$

Especially we can write

$$
R^{N_{0}}(x, \xi)=1+R(x, \xi) \quad \text { if } \xi \in V_{0}, \quad|\xi| \geq B+1,
$$

where $R(x, \xi)$ satisfies the above condition (F) with $m_{+}$replaced by $m_{+}(\rho-\delta) N_{0}$. Thus we can finally deduce the problem to the following equation of the form

$$
u+R_{1}(x, D) u=f,
$$

where $u \in \mathscr{E}^{\{\theta\}^{\prime}}(\Omega), f \in \mathscr{D}^{\{\theta\}^{\prime}}(\Omega)$ and $\left(x_{0}, \xi_{0}\right) \notin W F_{\{\theta\}}(f)$. We may assume $R_{1}(x, \xi)$ satisfies (F) with $m_{+}=-2 n-1$. As in the proof of [17], Theorem 2.2, localize the problem so that supp $u \subset\left\{x ;\left|x-x_{0}\right|<\varepsilon\right\}$. Take $\chi \in \mathscr{D}^{\{\theta\}}(\Omega)$ such that $\chi=1$ if $\left|x-x_{0}\right|<\varepsilon$ and $\chi=0$ if $\left|x-x_{0}\right|>2 \varepsilon$. Then the equa- 
tion (2.29) is written as

$$
u+\chi R_{1}(x, D) u=\chi f .
$$

It was proved in [17] that we have

$$
u=\left(I+\chi R_{1}\right)^{-1} \chi f=\sum_{j=0}^{\infty}\left(-\chi R_{1}\right)^{j}(\chi f)
$$

for sufficiently small $\varepsilon>0$. Since the condition $(F)$ yields the condition (1) and (2) of [24] with $\kappa=\sigma /(1-\delta)$, the results of [24] can be applied to the Neumann series $\sum_{j=0}^{\infty}\left(-\chi R_{1}\right)^{j}$. According to [24], this is represented as the sum $R_{2}(x, D)+R_{3}(x, D)$ of the pseudodifferential operators with symbols $R_{2}(x, \xi)$ and $R_{3}(x, \xi)$ where $R_{2}(x, \xi)$ satisfies the condition (1) and (2) of [24] with $\kappa=\sigma /(1-\delta)$ and $R_{3}(x, D)$ is a regularizer from $\mathscr{E}^{\{\theta\}^{\prime}}$ to $\mathscr{E}^{\{\theta\}}$. Again we apply the local formula of the form (2.4) for $R_{2}(x, D) \chi f$ and we can see $\left(x_{0}, \xi_{0}\right) \notin W F_{\{\theta\}}(u)$ under the condition $\left(x_{0}, \xi_{0}\right) \notin W F_{\{\theta\}}(\chi f)$.

Remark. The details of the proof of the results of [24] will be given in a colaboration of Shinkai and Taniguchi, [21].

\section{REFERENCES}

[1] Aronszajn, N., Preliminary notes for "Traces of analytic solutions of the heat equation" and Traces of analytic solutions of the heat equation, Colloque International C.N.R.S. sur les équations aux dérivées partielles linéaires, 2-3 (1973), $5-68$.

[2] Bony, J. M., Equivalence des diverses notions de spectre singulier analytique, Séminaire Goulaouic-Schwartz 1976-1977, Exposé No. 3.

[ 3 ] Bros, J. and D. Iagolnitzer, Support essentiel et structure analytique des distributions, Séminaire Goulaouic-Lions-Schwartz 1975, Exposé No. 18.

[4] Gel'fand, I. M. and G. E. Shilov, Generalized functions, Vol. 2, Academic press, New York-London, 1968.

[5] Hashimoto, S., T. Matsuzawa and Y. Morimoto, Opérateurs pseudodifferentiels et classes de Gevrey, Comm. Partial Differential Equations, 8 (12) (1983), 1277-1289.

[6] Hörmander, L., Pseudodifferential operators and hypoelliptic equations, Proc. symposium on singular integrals, Amer. Math. Soc., 10 (1967), 138-183.

[ 7 ] - The analysis of linear partial differential operators, Vol. 1, Springer-Verlag, Berlin-Heidelberg-New York-Tokyo, 1983.

[8] Iwasaki, C., Gevrey-hypoellipticity and pseudo-differential operators on Gevrey class, Lecture Notes in Math. Vol. 1256, Springer-Verlag, Berlin-Heidelberg-New York-Tokyo, 1986, 281-293.

[ 9 ] Kajitani, K. and S. Wakabayashi, Hypoelliptic operators in Gevrey classes, to appear.

[10] Kashiwara, M., Introduction to the theory of hyperfunctions, Sem. on microlocal analysis, Princeton Univ. Press, Princeton, N. J., 1979, 3-38.

[11] Kawai, T. and T. Matsuzawa, On the boundary value of the solution of the heat equation, to appear in Publ. RIMS, Kyoto Univ., 25 (1989). 
[12] Komatsu, H., Ultradistributions I; Structure theorems and a characterization, J. Fac. Sci. Univ. Tokyo, Sect. IA, 20 (1973), 25-105.

[13] - - Ultradistributions II ; The kernel theorem and ultradistributions with support in a submanifold, J. Fac. Sci. Univ. Tokyo, Sect. IA, 24 (1977), 607-628.

[14] - Introduction to the theory of generalized functions (in Japanese), Iwanami Shoten, 1978.

[15] Martineau, A., Les hyperfonctions de M. Sato, Séminaire Bourbaki 1960-1961, Exposé No. 214.

[16] Matsuzawa, T., Gevrey hypoellipticity of a class of pseudodifferential operators, Tôhoku Math. J., 39 (1987), 447-464.

[17] — Hypoellipticity in ultradistribution spaces, J. Fac. Sci. Univ. Tokyo, Sect. IA, 34 (1987), 779-790.

[18] — A calculus approach to hyperfunctions I, Nagoya Math. J., 108 (1987), 53-66.

[19] _- A calculus approach to hyperfunctions II, Trans. Amer. Math. Soc., 313, No. 2 (1989), 619-654.

[20] Miyake, M., Solvability of systems of ordinary defferential equations in the space of Aronszajn and the determinant over the Weyl algebra, to appear.

[21] Shinkai, K. and K. Taniguchi, Fundamental solution for a degenerate hyperbolic operator in Gevrey classes, to appear.

[22] Sjöstrand, J., Propagation of analytic singularities for second order Dirichlet problems, Comm. Partial Differential Equations, 5(1) (1980), 41-94.

[23] —- Singularités analytiques microlocales, Astérisques, 95 (1982), 1-166.

[24] Taniguchi, K., On multi-products of pseudodifferential operators in Gevrey classes and its application to Gevrey hypoellipticity, Proc. Japan Acad., Ser. A (1985), 291-293.

[25] Treves, F., Introducton to pseudodifferential and Fourier integral operators, Vol. 1, Plenum Press, New York-London, 1981.

Department of Mathematics

Meijo University

Tenpaku-ku, Nagoya 468

Japan 